\title{
Heterogenität in den Voraussetzungen beruflicher Orientierung
}

Im vorausgegangenen Kapitel hat sich gezeigt, dass die Berücksichtigung der individuellen Bedarfe, die aus der Heterogenität der Lernenden in Bezug auf ihre Voraussetzungen und Entwicklungsstände resultiert, eine der wesentlichen Herausforderungen in der Umsetzung erfolgreicher beruflicher Orientierung im Kontext von Schule darstellt. Um sich dem Anliegen dieser Arbeit zu nähern, widmet sich das folgende Kapitel daher den divergierenden persönlichen Voraussetzungen, die als eine der Ursachen der Heterogenität in der beruflichen Orientierung bewertet werden können.

Das erste Teilkapitel (3.1) fokussiert dabei die begriffliche Definition von Heterogenität, insbesondere in Abgrenzung zu Inklusion und Diversität. Im zweiten Teilkapitel (3.2) wird die Heterogenität im Berufswahlverhalten junger Menschen aufgrund unterschiedlicher persönlicher Voraussetzungen anhand von drei theoretischen Konstrukten rekonstruiert. Boudons (1974) Theorie der primären und sekundären Herkunftseffekte liefert eine Erklärung der sozial bedingten Chancenungleichheiten im Schul- und Bildungssystem. Diese theoretischen Überlegungen in Hinblick auf Chancenungleichheit werden zudem durch empirisch belegbare Unterschiede im Bildungserfolg bei Jugendlichen aus privilegierten und sozial benachteiligten Familien, bildungsnahen und -fernen Familien, sowie von Jugendlichen mit und ohne Migrationshintergrund sichtbar (OECD, 2014; Stanat et al., 2002). Mittels primärer und sekundärer Herkunftseffekte lassen sich jedoch nicht nur die Chancenungleichheiten im Schulsystem erklären. Boudons Theorie leistet auch einen wichtigen Beitrag, um sozial bedingte Unterschiede im Berufswahlverhalten zu erklären (Brändle \& Grundmann, 2013). Gottfredsons (1981) Theorie der Eingrenzung und Kompromissbildung erweitert Boudons These der klassenabhängigen Wert-Nutzen-Abschätzungen von Bildung durch ihren theoretischen Einbezug des Abgleichs der Jugendlichen zwischen 
ihrem eigenen sozialen Status und dem Prestige des Wunschberufs. Leistungsvergleichsstudien konnten neben sozialen Unterschieden zudem Abweichungen im Schulerfolg und den Aspirationen von Jungen und Mädchen nachzeichnen (OECD, 2015). Gottfredsons Ausführungen liefern ein theoretisches Fundament für diese geschlechtsbezogenen Differenzen in der Berufswahl (Gottfredson, 2002). Auch ihre theoretischen Überlegungen werden mit aktuellen empirischen Befunden verschränkt. Mit der dritten Theorie, nämlich Bourdieus (1982, 1987) Ausführungen zu milieugebundenem Habitus, werden Boudons (1974) und Gottfredsons (1981) Betrachtungen sozial- und geschlechtsbedingter Berufswahl um den Einfluss sozialisationsbedingter Distanzen zwischen Individuum und Beruf ergänzt.

Zusammenfassend wird am Ende dieses Kapitels dargestellt, dass sowohl die begriffliche Einordnung als auch die theoretische Verschränkung der heterogenitätsbezogenen Befunde dazu beiträgt, erste Konsequenzen auf sozial-, milieu- und geschlechtsbedingtes Berufswahlverhalten abzuleiten und für die forschungsseitige Unterstützung bedarfsbezogener beruflicher Orientierung nutzbar zu machen.

\subsection{Begriffsklärung}

Bevor die Heterogenität der Lernenden in ihrer beruflichen Entwicklung theoretisch und empirisch beleuchtet und damit Schlussfolgerungen über den Bedarf individualisierter Angebote beruflicher Orientierung gezogen werden können, bedarf es einer begrifflichen Herleitung.

Für eine begriffliche Einordnung von Heterogenität ist zunächst anzumerken, dass keine allgemeingültige Definition existiert, sondern vielmehr eine Vielfalt an sich überschneidenden Beschreibungen besteht. Einen Überblick dazu gibt Scharenberg (2013). Heterogenität beschreibt in seiner breitesten Definition die „Differenz zwischen wenigstens zwei Elementen“ (Budde, 2015, S. 23). Im Kontext empirischer Bildungsforschung kann Heterogenität als die ,unterschiedlichen Lernvoraussetzungen" (S. 1) verstanden werden, die sich u. a. aus dem Geschlecht, dem Alter, der sozialen und kulturellen Herkunft, der Sozialisation sowie den kognitiven Fähigkeiten und der motivationalen Bereitschaft ergeben (Scharenberg, 2013). Budde (2015) kritisiert in Bezug auf die Beschreibung von Heterogenität eben diese zunehmend weite Fassung, da sie wiederum die Operationalisierung des Begriffs für die Forschung erschwert. Gleichzeitig betont er, dass Heterogenität, Homogenität und Individualität ein Spannungsfeld 
ergeben, dass als Ganzes gedacht und interpretiert werden muss. Schulleistungsstudien, wie PISA (OECD, 2014), zeigen die Zusammenhänge zwischen den heterogenen Voraussetzungen der Lernenden und ihren Leistungsergebnissen auf. Diese Unterschiede in den Lernvoraussetzungen werden anhand von festgelegten Standards beschrieben und eingeordnet. Entsprechend stellt Heterogenität auch ein auf Homogenität basierendes ,normatives Phänomen“ (Scharenberg, 2013, S. 5) dar. Insofern sind Leistungstests auf der einen Seite zugleich Mittel und Zweck einheitlicher und vergleichbarer Standards. Auf der anderen Seite adressieren didaktische Konzepte, wie das jahrgangsübergreifende Lernen in der Schuleingangsphase, der fächerübergreifende Unterricht oder die leistungsbezogene Binnendifferenzierung, die heterogenen Voraussetzungen der Jugendlichen und damit ihre Individualität (Budde, 2015). Das bipolare Spannungsverhältnis zwischen Heterogenität und Homogenisierung manifestiert sich selbst innerhalb eines didaktischen Konzepts. So kann die Zusammenfassung von Jugendlichen mit ähnlichen Voraussetzungen bzw. Bedarfen im Kontext der unterrichtsbezogenen Binnendifferenzierung als Bemühung der Homogenisierung, oder aber als Berücksichtigung von Individualität und der damit in Verbindung stehenden Heterogenität interpretiert werden (Budde, 2015). Die Schaffung von Vergleichbarkeit, die Betonung von Differenzen und die gleichzeitige Berücksichtigung individueller Bedarfe stellen für Budde (2013) infolgedessen ein relationales Kraftfeld dar. Für die Umsetzung von Chancengleichheit im schulischen Kontext bedeutet Heterogenität, wie Budde, Blasse, Bossen und Rißler (2015) betonen, zugleich Chance und Herausforderung. Heterogenität wird in verschiedenen Modellen u. a. von Wenning (2007) und Heyer, Preuss-Lausitz und Sack (2003) als soziales Phänomen erfasst, in Konsequenz dessen Unterschiede der Lernenden im sozialen Kontext betrachtet werden (vgl. auch Scharenberg, 2013).

Heterogenität lässt sich zudem in Abgrenzung zu dem Begriff der Diversity - der englische Terminus für Diversität ist in den deutschen Sprachgebrauch übergegangen - sowie zu den Begriffen Inklusion und Integration erklären. Diversity beschreibt ein Verständnis von Differenz, bei dem kein spezifisches Merkmal, wie der Migrationshintergrund oder das Geschlecht, im Vordergrund steht (Plößner, 2013). Vielmehr versteht Diversity Differenz als das Ergebnis einer Fülle an Kombinationsmöglichkeiten vielfältiger Differenzmerkmale (Plößner, 2013). In Abhängigkeit der Perspektive kann Diversity in unterschiedlichster Art, beispielsweise als positives Grundverständnis von Heterogenität, als Sensibilisierungsmöglichkeit für Unterschiede, als Ansatzpunkt für Gerechtigkeit, aber auch als zu managendes Kriterium wirtschaftlicher Gewinnmaximierung verstanden werden. Ihnen gemeinsam ist die Pluralität der Verschiedenheit (Plößner, 2013). 
Die Begriffe Heterogenität und Diversity überschneiden sich folglich. Mit der Verwendung des Begriffs der Heterogenität liegt der Schwerpunkt jedoch stärker auf der Feststellung und Beleuchtung von Unterschieden, während mit dem Begriff Diversity ein stärkerer Fokus auf die Einstellungen zu diesen Unterschieden unternommen wird (Plößner, 2013).

Auch den Begriffen Inklusion und Integration liegt kein einheitliches Begriffsverständnis zugrunde (Grosche, 2015), was ihre Operationalisierung und Erforschung im bildungswissenschaftlichen Kontext ähnlich wie die der Heterogenität erschwert. Grosche (2015) argumentiert, dass die deutschsprachigen Begriffe Inklusion und Integration mit Blick auf die Begriffsverwendung und -übersetzung der Salamanca-Erklärung (UNESCO, 1994) synonym verwendbar sind. Inklusion wird häufig als die gemeinsame Beschulung von Lernenden mit und ohne Behinderung verstanden (Grosche, 2015). Jedoch greift diese Definition nach Einschätzung von Grosche (2015) zu kurz, denn ähnlich den Begriffen der Heterogenität und der Diversity beschreibt Inklusion verschiedene Dimensionen und Ebenen von Unterschieden im gesellschaftlichen Kontext und kann in Abhängigkeit der Perspektive sowohl für Einstellungen zu Vielfalt als auch für die sich aus Vielfalt ergebenden bzw. erstrebenswerten gesellschaftlichen Veränderungsprozesse stehen. Ainscow, Booth und Dyson (2006; zit. n. Grosche, 2015) sehen Inklusion entsprechend als ,die Anwesenheit, Teilhabe und die Leistung aller [aktuell ausgegrenzten] Lernenden“ (Grosche, 2015, S. 20) sowie den restrukturierenden Prozess, der im Kontext von Schule, Kultur und Gesellschaft notwendig ist, „um der Heterogenität aller Lernenden gerecht zu werden“ (Grosche, 2015, S. 21).

Unter Berücksichtigung des zuvor erläuterten Begriffs der Diversity, der die Verschiedenheit aller Menschen betont, soll in dieser Arbeit Heterogenität als die Zusammenfassung aller Merkmale, über die sich Jugendliche in ihrer beruflichen Entwicklung unterscheiden, verstanden werden. Inklusion wiederum wird als die Summe der Bemühungen zur Ansprache heterogener beruflicher Entwicklungsbedarfe und zur Sicherung der beruflichen Orientierung gemäß den individuellen Möglichkeiten verstanden. Im Folgenden werden ausgewählte theoretische Konzepte und empirische Befunde zu Ursachen berufswahlbezogener Heterogenität Jugendlicher dargestellt und anschließend sich daraus ableitende Forschungsdesiderata benannt. 


\subsection{Theoretische Betrachtungen heterogener Ausgangslagen beruflicher Orientierung}

Der Einfluss individueller Faktoren wie dem Geschlecht, dem sozioökonomischen und kulturellen Hintergrund auf das Berufswahlverhalten junger Menschen wurde vielfach empirisch beleuchtet (Beicht \& Walden, 2019; Driesel-Lange, 2011; Heublein et al., 2017) und im wissenschaftlichen Diskurs erörtert (Brändle \& Grundmann, 2013; Jordan \& Kauffeld, 2019; Patton \& McMahon, 2014). Das folgende Teilkapitel präsentiert drei theoretische Konzepte, die die in der sozialen Herkunft und Sozialisierung bestehenden Unterschiede zwischen Jugendlichen zur Erklärung differierenden Berufswahlverhaltens heranziehen. Boudons Erläuterungen zu primären und sekundären Herkunftseffekten werden durch Bourdieus Habituskonzept (1982) als Fundament milieugebundener Berufswahl und schließlich Gottfredsons (1981, 2002) Überlegungen zum Abgleich zwischen dem eigenen Selbst und der geschlechts- und prestigebezogenen Wahrnehmung des Berufs ergänzt. Die drei Theorien werden zunächst einzeln vorgestellt und in ihrer Relevanz für die Erklärung heterogenen Berufswahlverhaltens unter Verschränkung mit der aktuellen empirischen Befundlage diskutiert.

\section{Boudons Theorie der primären und sekundären Herkunftseffekte}

Nach Boudon (1974) können Unterschiede in Bildungsverläufen durch primäre und sekundäre Herkunftseffekte erklärt werden. Damit baut Boudon auf den Ausführungen von Keller und Zavalloni (1962, 1964, zit. n. Brändle \& Grundmann, 2013) zu den Zusammenhängen zwischen der sozialen Herkunft und Ambitionen auf. Primäre Herkunftseffekte werden von Boudon als die direkten Auswirkungen der sozialen Herkunft, vor allem dem sozialen Status der Familie und der gleichsamen Sozialisierung, auf die schulischen Leistungen von Heranwachsenden verstanden (Boudon, 1974; vgl. auch Relikowski, Schneider \& Blossfeld, 2010). Mit steigendem sozialen Status einer Familie erhöht sich auch ihr Bildungs- und kulturelles Kapital. Die damit wachsenden Unterstützungsmöglichkeiten der schulischen Lernprozesse der Kinder schlagen sich wiederum in besseren schulischen Leistungen nieder (Boudon, 1974). Die von Boudon beschriebenen Herkunftseffekte konnten vielfach durch nationale und internationale Schulleistungstests, wie PISA (OECD, 2014), belegt werden. Kinder aus sozial benachteiligten Familien verfügen aufgrund ihres sozialen Hintergrunds über geringere Chancen, in der Schule erfolgreich zu sein (Zielonka et al., 2013). Auch auf die beruflichen Verläufe und Erfolge wirkt sich die soziale Herkunft als entsprechender Primäreffekt aus. Im Vergleich zu jenen mit einem niedrigen Sozialstatus erwerben Menschen, die aus Familien mit einem höheren Sozialstatus stammen, eher 
einen höheren Bildungsabschluss, der, Boudons Theorie weiterführend, wiederum zu einem prestigeträchtigeren und/oder besser bezahlten Beruf führt (Brändle \& Grundmann, 2013).

Neben den primären Herkunftseffekten erklären sekundäre Herkunftseffekte gemäß Boudon (1974) „die Reproduktionslogiken von Bildungsungleichheiten“ (Brändle, S. 62). Unter Sekundäreffekten versteht Boudon (1974) sozialstatusbedingte Unterschiede im Entscheidungsverhalten. Im Konkreten werden sowohl die Kosten von Bildung, im Sinne monetärer und sozialer Anstrengungen, als auch der wahrgenommene und erwartete Nutzen von Bildung in Abhängigkeit des sozialen Status unterschiedlich bewertet (Relikowski et al., 2010). Eltern mit einem hohen Sozialstatus verfügen bereits über vergleichbar große Ressourcen zur Bildungsförderung ihrer Kinder. Dazu zählen u. a. ihr Wissen über das Schulund Übergangssystem oder ihr berufliches Netzwerk. Entsprechend gering sind die Kosten einer höheren Bildung für ihre Kinder (Brändle \& Grundmann, 2013). Gleichzeitig steigt der erwartete Nutzen von Investitionen in höhere Bildung durch ihre höheren Erfolgschancen im Bildungssystem und die darauf aufbauenden besseren Berufschancen (Brändle \& Grundmann, 2013).

Diese ,schichtspezifische Bildungsrationalität“ (Brändle \& Grundmann, 2013, S. 62) basiert in erster Linie auf dem Bestreben, den Status durch die nächste Generation entweder zu erhalten oder zu verbessern (Brändle \& Grundmann, 2013). Entsprechend des hohen Sozialstatus der eigenen Familie, den es zu erhalten gilt, entwickeln diese Jugendlichen nach Boudons Theorie auch höhere berufliche Ambitionen als Jugendliche aus sozial benachteiligten Familien. Für Jugendliche aus sozial benachteiligten Familien stellen Bildungs- und Berufsentscheidungen zwar Möglichkeiten der Statusverbesserung dar, allerdings wird der Nutzen höherer Bildung aufgrund der eigenen geringeren Bildungschancen wiederum niedriger eingeschätzt (Brändle \& Grundmann, 2013). Denn die Chancen eines erfolgreichen Schulabschlusses und einer sich anschließenden höheren beruflichen Laufbahn bleiben aufgrund der Primäreffekte hinter denen der hohen Sozialstatusgruppe zurück. Gleichzeitig scheinen die Kosten höherer Bildung und prestigeträchtiger Berufe für Jugendliche aus sozial benachteiligten Familien höher (Brändle \& Grundmann, 2013). Gründe dafür liegen u. a. in der größeren sozialen Distanz zwischen dem angestrebten Beruf und der Herkunftsfamilie. Die Überbrückung dieser sozialen Distanz erfordert größere Anstrengungen und wird beispielsweise durch ein geringeres Bildungskapital der Eltern erschwert, das sich in Informationsdefiziten hinsichtlich des Übergangs- oder Hochschulsystems manifestieren kann (Relikowski et al., 2010). Aufgrund des individuell 
rational ungünstigeren Kosten-Nutzen-Verhältnisses entwickeln sozial benachteiligte Jugendliche entsprechend geringere berufliche Ambitionen (Brändle \& Grundmann, 2013).

Mit dieser Unterscheidung zwischen Primär- und Sekundäreffekten beschreibt Boudon die Mehrdimensionalität der Wirkmechanismen von sozialer Herkunft auf Bildungs- und Berufschancen (vgl. Relikowski et al., 2010). Weiter zeigt er anhand der Sekundäreffekte auf, dass Menschen zwar individuell rationale Entscheidungen in Bezug auf ihre Bildungsinvestitionen treffen, diese Entscheidungen aber bereits aufgrund der eigenen sozialen Herkunft in einem eingeschränkten Handlungsrahmen stattfinden. Auf die berufliche Orientierung übertragen bedeutet das, dass Jugendliche zwar einerseits durch ihren Bildungsabschluss, der wiederum durch ihre soziale Herkunft beeinflusst wird, in ihrer Berufswahl eingeschränkt sind, sie darüber hinaus aber selbst aufgrund einer statusbedingten Kosten-Nutzen-Abwägung das Feld der anzustrebenden Berufe eingrenzen (Brändle \& Grundmann, 2013).

\section{Bourdieus Habitus Theorie}

Mit Bourdieus Habitustheorie werden Boudons (1974) sozialtheoretische Betrachtungen sozial bedingter Berufswahl um den Einfluss sozialisationsbedingter Distanzen zwischen Individuum und Beruf ergänzt.

Als Habitus werden die bereits in der frühen Kindheit und Jugend durch Sozialisierungsprozesse erworbenen und verinnerlichten Vorlieben, Haltungen und Verhaltensweisen einer Person bezeichnet (Scherr, 2016). Diese finden u. a. in der Sprache oder dem Erscheinungsbild eines Individuums Ausdruck. Nach Bourdieu (1982) sind diese Verhaltensweisen nicht nur, wie bereits von Elias (1994) festgestellt, vielseitig und vielschichtig, sondern auch spezifisch für soziale Milieus und Klassen (Liebsch, 2016). Entsprechend lässt der Habitus Rückschlüsse auf den sozialen Status bzw. die Zugehörigkeit zu einem spezifischen sozialen Milieu zu. Weiter stellt der Habitus, so Bourdieus Annahme, eine Voraussetzung zur Interaktion und Partizipation in einer spezifischen Gesellschaftsschicht dar (Bourdieu, 1982).

In der Prägung des Habitus stellen das ökonomische, kulturelle, soziale und symbolische Kapital zentrale Faktoren dar (Liebsch, 2016; Thieme, 2016). Unter ökonomischem Kapital ist im Kontext der beruflichen Entwicklung Jugendlicher die Gesamtheit der materiellen Mittel der Eltern und der Familie zu verstehen. Das kulturelle Kapital entsteht besonders durch den Bildungsstand der Eltern, aber auch durch die Art und Intensität des Kultur- und Medienkonsums der Familie (Liebsch, 2016). Das soziale Kapitel umfasst die sozialen Ressourcen der Familie wie beispielsweise ihre Netzwerke (Thieme, 2016). Im Zusammenhang mit der 
Berufswahl stellen beispielsweise die beruflichen Verbindungen der Eltern, die zur Erlangung eines Praktikumsplatzes oder zur Informationsakquise genutzt werden können, relevantes soziales Kapital dar. Das symbolische Kapital spiegelt den sozialen Status und die Anerkennung im eigenen sozialen Raum wider (Thieme, 2016).

Nach Bourdieu stellt der Habitus ein Resultat bestehender, durch das Individuum inkorporierter Strukturen dar, die auch als opus operatum bezeichnet werden (Bourdieu, 1976; Liebsch, 2016). Zeitgleich bildet er die grundlegende Perspektive, aus der heraus die eigene Umwelt wahrgenommen und interpretiert wird und aus der entsprechende Handlungsweisen (modus operandi) entstehen. Dadurch werden die bestehenden sozialen Strukturen wiederum reproduziert (Bourdieu, 1976; Bourdieu \& Wacquant, 1996).

Bourdieus Annahme, dass der Habitus aufgrund der Lern- und Erfahrungsprozesse ein stabiles Konstrukt darstellt, das nur sukzessive, beispielsweise durch prägende Ereignisse veränderbar ist (Liebsch, 2016), ist für das Verständnis der Gelingensbedingungen erfolgreicher beruflicher Orientierung hochgradig bedeutsam. So treffen Jugendliche aus verschiedenen sozialen Schichten entsprechend ihrer differierenden habituellen Dispositionen eine ,milieugebundene Berufswahl“ (Brändle \& Grundmann, 2013, S. 64). Diese basiert auf beruflichen Aspirationen und Explorationen. Beide beschränken sich mehrheitlich auf ein Berufsfeld, das dem eigenen sozialen Milieu hinsichtlich des Berufsprestiges entspricht und im eigenen sozialen Milieu beobachtete berufliche Tätigkeiten enthält (Brändle \& Grundmann, 2013). Die sogenannten intergenerationalen Transmissionen (Busch, 2013), also die Reproduktion bzw. „Vererbung“ von Bildungsstand und Beruf der Eltern an ihre Kinder, lassen sich folglich (auch) anhand Bourdieus Habitustheorie erklären.

Eine zweite Schlussfolgerung, auf die in der Anwendung des Habituskonzepts auf die berufliche Orientierung verwiesen werden soll, bezieht sich auf die Bedarfe, die aus einer milieufremden Berufswahl entstehen. Wählen Jugendliche einen Beruf, der außerhalb ihres eigenen sozialen Milieus liegt, bewegen sie sich in einem neuen sozialen Feld, dessen Habitus nicht dem eigenen entspricht und sozusagen unbekanntes Terrain darstellt. Dies lässt sich plastisch am Beispiel eines Jura studierenden „Arbeiterkinds“ wie folgt darstellen: Mit dem Berufswunsch Richter*in aus einer Familie mit nichtakademischem Hintergrund stammend, erlebt die Person bereits zu Beginn des Jurastudiums ein neues Umfeld mit einem ihr/ihm unbekannten Habitus. Ihr/ihm sind die Sprachweisen, der Kleidungsstil, die Rituale oder unausgesprochenen Regeln, die zwischen den angehenden Jurist*innen bestehen, (zunächst) unbekannt. Der Habitus des neuen sozialen Umfelds kann nun anhand der kulturellen Praktiken erworben 
und erlernt werden (Liebsch, 2016). Der Habitus kann folglich in Teilen oder gänzlich, beispielsweise durch die Verwendung der gleichen Redewendungen oder durch die Anpassung der eigenen Garderobe, angenommen werden. Der oder die Studierende kann sich jedoch auch bewusst - möglicherweise mit dem Risiko einer weniger erfolgreichen Laufbahn - von den unter Jurist*innen gängigen Gepflogenheiten abgrenzen und diese nicht für sich selbst annehmen. Für diese Entscheidungen bedarf es jedoch der vorherigen Reflektion der zu erwartenden sozialen, kulturellen und ökonomischen Veränderungen, die die eigene Berufswahl begleiten werden, um einem „Kulturschock“ entgegenzuwirken und in einer längerfristigen Perspektive habituell adäquates Verhalten annehmen zu können. Daraus lässt sich das Antizipationsvermögen als eine bedeutende Gelingensbedingung erfolgreicher beruflicher Übergänge ableiten. Im Thüringer Berufswahlkompetenzmodell, das in Kapitel 6 ausführlich vorgestellt wird und das den empirischen Studien in Kapitel 10, 11 und 12 zugrunde liegt, wird dieser Antizipationsprozess als eine phasentypische Entwicklungsaufgabe der letzten Berufswahlphase verstanden (Driesel-Lange et al., 2020).

\section{Gottfredsons Berufswahltheorie der Eingrenzung und Kompromissfindung}

Gottfredson (1981) setzt mit ihrer Theorie der Eingrenzung und Kompromissfindung ähnlich wie Boudon (1974) an der sozialen Herkunft als Faktor der Berufswahl an. Jedoch ergänzt sie diesen durch eine entwicklungspsychologische Perspektive, in der sie auch die Relevanz von Prestige und den Einfluss des Geschlechts auf die Berufswahl sowie die Entwicklung von Interessen integriert ${ }^{1}$. Wie im Folgenden ausdifferenziert wird, fokussiert Gottfredson im Gegensatz zu Boudon nicht den durch den Sozialstatus bedingten Kosten-Nutzen-Abgleich im

\footnotetext{
${ }^{1}$ Auch die Humankapitaltheorie und die Sozialisationstheorie bieten - wie Gottfredson - theoretische Erklärungen für geschlechtsbezogene Berufswahlunterschiede. Die Humankapitaltheorie von Becker (1975, zit. n. Busch, 2013) erklärt die geschlechtsspezifische Berufswahl durch geschlechtsspezifische Kosten-Nutzen-Abwägungen. Demnach wägen (junge) Frauen Bildungsinvestitionen mit Blick auf später ausfallende Erträge durch Familienzeiten stärker $a b$ und orientieren sich mehrheitlich in Richtung von Berufen, die mit den Bedürfnissen der zu gründenden Familien in Einklang zu bringen sind (Busch, 2013).

Die Sozialisationstheorie von Beck-Gernsheim und Ostne (1978, zit. n. Busch, 2013) wiederum erklärt das zwischen Männern und Frauen differierende Berufswahlverhalten durch „geschlechtsspezifische Präferenzen von Arbeitsinhalten“ (Busch, 2013, S. 149). Aufgrund der Fokussierung dieser Arbeit auf die individuellen Bedarfe in der beruflichen Orientierung sollen diese beiden angebotsseitig orientierten Theorien jedoch an dieser Stelle nur in Kürze erwähnt und nicht weiter ausgeführt werden. Einen ausführlichen Überblick bietet Busch (2013).
} 
Berufswahlprozess (Brändle \& Grundmann, 2013). Vielmehr beschreibt Gottfredson den Entscheidungs- und Berufswahlprozess als eine entwicklungsbedingte Eingrenzung beruflicher Aspirationen im Kindheits- und Jugendalter (1981, 1996, 2002). Dieser Eingrenzungsprozess findet insbesondere über einen Kompatibilitätsabgleich des Selbstbilds, einem Produkt des eigenen Sozialstatus und der Geschlechtsidentität, mit dem Berufsbild statt (Gottfredson, 2002). Das Berufsbild spiegelt die stereotype Wahrnehmung des Berufsprestiges sowie seiner Geschlechtskonnotation in der Gesellschaft (Gottfredson, 2002). Damit verbunden sind unter anderem die (vermeintlich) mit dem Beruf einhergehenden Tätigkeiten, das berufliche Umfeld und Bedingungen, aber auch Stereotypen der Persönlichkeiten und Lebensstile, derer, die diesen Beruf ausüben (Gottfredson, 2002). Aus den Vorstellungen des Berufs, vor allem aus dem intellektuellen Anspruch der Tätigkeiten, lässt sich weiter das gesellschaftliche Ansehen des Berufs (Berufsprestige) ableiten. Aus dem Eindruck der in diesem Beruf tätigen Personen entsteht zudem eine innerhalb einer Gesellschaft einheitliche Geschlechtszuordnung eines Berufs, also die Vorstellung, ob ein Beruf eher von Frauen oder Männern ausgeübt wird (Brändle \& Grundmann, 2013).

Heranwachsende ordnen Berufe entsprechend ihrer Geschlechtskonnotation und ihres Prestiges auf einer im sozialen Kontext allgemeingültigen Landkarte an. Gleichzeitig grenzen sie die für sich selbst in Frage kommenden Berufe über die Abschätzung der Kompatibilität des Berufs in Hinsicht auf sein Prestige und seine Geschlechtskonnotation mit der eigenen Geschlechtsidentität und dem eigenen Sozialstatus sukzessive ein. Je höher die Kompatibilität des Berufsbilds mit dem Selbstbild, desto höher die Aspiration des Berufs. Auf der zuvor genannten Landkarte entsteht ein Bereich der beruflichen Aspirationen. Dieses Aspirationsfeld, in dem die weitere Suche stattfindet, ist nach oben und unten durch den Prestigegrad begrenzt, der hinsichtlich des Berufsprestige-Sozialstatus-Abgleichs gerade noch realistisch erreichbar (akzeptabel) ist. Nach links und rechts begrenzt sich das Suchfeld durch Berufe, deren Geschlechtskonnotation gerade noch mit dem eigenen Selbstbild vereinbar ist (Gottfredson, 2002).

Der Eingrenzungsprozess verläuft beginnend ab der frühen Kindheit in vier zentralen Phasen, die durch kognitive und entwicklungsbedingte, individuelle Unterschiede geprägt sind und nach Gottfredson (2002) wie folgt zusammengefasst werden können:

(1) Orientierung an Größe und Macht (3.-5. Lebensjahr)

- Erfassung geschlechtstypischer Unterschiede

- Erkennen von Arbeit und Berufen als Teil der Erwachsenenwelt

(2) Orientierung an Geschlechterrollen (6.-8. Lebensjahr) 
- Eigene Geschlechterrollenorientierung

- Geschlechtsbezogene Eingrenzung des beruflichen Aspirationsfelds

(3) Orientierung am sozialen Wertebereich (9.-13. Lebensjahr)

- Eigene Abgrenzung über den sozialen Status

- Prestige- und leistungsbezogene Eingrenzung des beruflichen Aspirationsfelds

(4) Orientierung am inneren, einzigartigen Selbst (ab dem 14. Lebensjahr)

- Erfassung und Auseinandersetzung mit persönlichen Werten, Interessen und Zielen

Neben der Geschlechtsidentität beeinflussen in der dritten Phase der soziale Status, die Herkunft, das Alter und die Leistungsfähigkeit der Jugendlichen den Prestigegrad des Berufswunschs (Gottfredson, 2002; Rahn, Brüggemann \& Hartkopf, 2014). Gottfredson verankert damit in ihrer Theorie die Intersektionalität der persönlichen Merkmale.

Der zweite zentrale Prozess in Gottfredsons Theorie ist der Abgleich zwischen den idealistischen Aspirationen der Jugendlichen und den für sie aufgrund diverser anforderungs- und aufwandsbezogener Faktoren erreichbar scheinenden beruflichen Optionen (1981, 2002). Es erfolgt sozusagen ein Abgleich des eigenen Wunschszenarios mit der Realität in Bezug auf formale Voraussetzungen, Verfügbarkeit von Ausbildungs- bzw. Studienplätzen, regionale Strukturen und die eigene Mobilität. Innerhalb dieser Kompromissfindung werden nach der Entwicklung konditioneller Prioritäten die bestehenden Optionen nach „gut genug“ bzw. „nicht gut genug“ eingeschätzt. Bei der Auswahl der beruflichen Szenarien steht jedoch die Sicherung des sozialen Ansehens vor der Verwirklichung des inneren Selbsts (Gottfredson, 2002).

Als Folge werden eher Kompromisse bei den beruflichen Tätigkeiten zugunsten einer geschlechts- und prestigebezogenen Passung akzeptiert. Die Wahrnehmung der Erreichbarkeit und Kompatibilität eines Berufs ist subjektiv und hängt insbesondere von der Art und dem Grad der Information des Individuums ab (Gottfredson, 2002). Am Ende dieses Prozesses verbleiben wenige konkrete Optionen bzw. eine finale Berufswahl (Gottfredson, 2002).

Wie bereits bei Boudon, handelt es sich auch bei den von Gottfredson beschriebenen Eingrenzungsprozessen um individuell rationale Entscheidungen, die jedoch durch hintergründig bestehende, aber individuell verkannte Mechanismen gesteuert werden (Brändle \& Grundmann, 2013). Gottfredson (2002) betont explizit die Fatalität dieses Eingrenzungsprozesses, der einsetzt bevor er verstanden wird und aufgrund seines schleichenden Charakters von den Jugendlichen 
ohne externe Impulse nicht wahrgenommen (Gottfredson, 2002) und konterkariert werden kann. Die Größe des Aspirationsfelds ist zudem von der individuellen Kombination der Faktoren Prestige und Geschlechtskonnotation abhängig (Gottfredson, 2002). Da Jugendliche nicht zwangsläufig wahrnehmen, dass dieser Eingrenzungsprozess in Teilen auf den gesellschaftlich konstruierten Faktoren der Geschlechtsrollenidentität und des sozialen Status beruht und damit das eigene Aspirationsfeld auch flexibel veränderbar wäre, explorieren sie ihre beruflichen Möglichkeiten nur innerhalb des eingegrenzten Feldes (Gottfredson, 2002). Das Aspirationsfeld wie auch der konkrete Berufswunsch können sich also in Abhängigkeit einer Wahrnehmungsveränderung von Berufseignung und -erreichbarkeit ändern (Gottfredson, 2002), beispielsweise ausgelöst durch gendersensible Angebote beruflicher Orientierung. Im folgenden Teilkapitel werden die empirischen Befunde zu den gerade theoretisch hergeleiteten heterogenen Voraussetzungen in den Bildungsverläufen sowie in der beruflichen Orientierung entfaltet.

\subsection{Empirische Befunde zu heterogenen Voraussetzungen}

\section{Herkunfts- und milieubedingtes Berufswahlverhalten}

Die herkunftsbedingten Chancenungleichheiten im Schulsystem sind durch internationale Leistungsvergleichsstudien vielfach belegt (Klieme et al., 2010; OECD, 2014; Stanat et al., 2002). Die sich darin manifestierenden geringeren Leistungen von Heranwachsenden aus sogenannt sozial schwächeren bzw. bildungsfernen Familien stellen nach Boudon (1974) primäre Herkunftseffekte dar. Insofern lohnt sich auch der Blick auf den direkten Zusammenhang zwischen Schulleistungen, als Ergebnis primärer Herkunftseffekte, und der beruflichen Orientierung. Gute Schulnoten begünstigen, insbesondere bei Hauptschüler*innen, einen erfolgreichen Ausbildungseintritt (Protsch \& Dieckhoff, 2011) und stehen darüber hinaus in einem positiven Zusammenhang mit der Entwicklung eines konkreten Berufswunschs (Schmude, 2010). Ein höherer Schulabschluss als Folge guter Schulleistungen wirkt sich zudem begünstigend auf den Übertritt von der Schule in die Ausbildung aus (Beicht \& Walden, 2019). Jedoch erwerben Heranwachsende aus nicht-akademisierten oder sozioökonomisch benachteiligten Familien im Durchschnitt deutlich seltener einen höheren Schulabschluss (Beicht \& Walden, 2019), erreichen durchschnittlich weniger gute Schulabschlussnoten (Schneider, Franke, Woisch \& Spangenberg, 2017) und erleben beim Übergang in das Ausbildungssystem mehr Schwierigkeiten (Beicht \& Walden, 2014). Gleiches trifft auf Heranwachsende mit Migrationshintergrund zu (Beicht \& Walden, 2019; Lörz, Quast \& Woisch, 2012; 
Relikowski et al., 2010; Schneider et al., 2017). Im internationalen Vergleich wiegen die Nachteile für Heranwachsende mit Migrationshintergrund in Deutschland besonders schwer (Relikowski et al., 2010). Aufgrund dessen ist daher auch in der Betrachtung der primären Herkunftseffekte ein intensiver Blick auf die empirische Befundlage zu Deutschland besonders relevant. Junge Migrant*innen trifft dabei zudem oftmals eine kumulative Benachteiligung, da Familien mit Migrationshintergrund überproportional häufig auch einen geringen Sozialstatus aufweisen (Relikowski et al., 2010).

Die soziale Ungleichheit durch primäre Herkunftseffekte zeigt sich nicht nur in den nachschulischen Übergangen, sondern auch in dessen weiteren Verlauf: Studierende aus akademisierten Familien schließen im Verhältnis häufiger ihr Studium ab (Heublein et al., 2017), während Personen mit Migrationshintergrund im Vergleich zur autochthonen Bevölkerung ein größeres Risiko tragen, dauerhaft ohne Berufsabschluss zu verbleiben (BIBB, 2019).

Neben den Schulleistungen zeigt sich die Vertrautheit der Eltern mit dem Bildungs- und Übergangssystem als Teil des kulturellen Kapitals nach Bourdieu auch empirisch als bedeutsamer Gelingensfaktor von Bildungslaufbahnen (Watermann, Daniel \& Maaz, 2014). Entsprechend konnte am Beispiel von Hauptschüler*innen auch ein signifikanter Zusammenhang zwischen der Bildungsnähe der Eltern und der berufswahlbezogenen Entwicklung und den Ausbildungschancen ihrer Kinder festgestellt werden (Kohlrausch \& Solga, 2012). Für Jugendliche mit Migrationshintergrund zeigt die empirische Befundlage ein differenziertes Bild. Beicht und Walden (2014) identifizieren die schlechtere Ressourcenausstattung der Migrantenfamilien, im Sinne von Bourdieus Sozialkapital, als eine der Ursachen für die geringeren Übergangschancen in die betriebliche Ausbildung. Andererseits konnten türkische Bildungsaufsteiger*innen in ihren Familien auf einen hohen ,generationsübergreifende[n] Erfahrungs- und Wissensvorrat“ (Tepecik, 2011, S. 261) zurückgreifen. Zwar entspricht dieses „migrantenspezifische Bildungskapital" (Tepecik, 2011, S. 304) aufgrund fehlender Bildungsabschlüsse oder Zertifikate nicht dem institutionalisierten oder objektiven kulturellen Kapital nach Bourdieu (1983, zit. n. Tepecik, 2011), dennoch wird es von den Jugendlichen als wertvolle Ressource für die eigene Bildung wahrgenommen (Tepecik, 2011).

Hinsichtlich der sekundären Herkunftseffekte zeigt sich wiederum ein diffuses Bild. Denn einerseits entscheiden sich jungen Menschen mit einem niedrigeren Sozialstatus gemäß Boudons (1974) Theorie seltener für die Aufnahme eines Studiums (Autorengruppe Bildungsberichterstattung, 2018) und schätzen ihre Erfolgsaussichten im Studium geringer ein als Studienberechtigte aus akademisierten bzw. sozial privilegierten Elternhäusern (Schneider et al., 2017). 
Andererseits erleben Letztere häufiger eine größere Unsicherheit hinsichtlich der nächsten nachschulischen Schritte (Schneider et al., 2017). Diese können aus mehr wahrgenommenen Optionen aufgrund des größeren Sozialkapitals der Familie, beispielsweise dem beruflichen Netzwerk der Eltern, entstehen oder aus weniger entwickelten Berufswahlkompetenzen resultieren ${ }^{2}$. Die Studienaufnahme wird neben den bereits genannten Faktoren zudem durch „die Erwartungen und Bedingungen des Elternhauses" (Lörz et al. 2012, S. 2) im Sinne sozial bedingter familiärer Kosten-Nutzen-Analysen und des Statuserhalts bzw. der Statusverbesserung beeinflusst. Das Motiv der Statusverbesserung nach Boudon (1974) wirkt sich auch auf die beruflichen Ziele der Jugendlichen aus. Studierende aus nicht-akademisierten Elternhäusern streben häufiger ein hohes Einkommen an (Schneider et al., 2017), während Studierende aus bildungsnahen Familien im Verhältnis häufiger in prestigeträchtigen Studiengängen und an Universitäten zu finden sind (Heublein et al., 2017; Schneider et al., 2017).

Auch für Migrant*innen spielt die Statusverbesserung bei der Ausbildungsund Berufswahl eine zentrale Rolle (Tepecik, 2011), jedoch lassen sich die milieubedingten Sekundäreffekte nicht exakt empirisch rekonstruieren. Denn zum einen konnten Relikowski et al. (2010) keine Effekte sozialer Distanz bei den Bildungsentscheidungen von sozial benachteiligten Familien mit Migrationshintergrund feststellen, im Gegensatz zu denen autochthoner Familien. Zum anderen scheint die herkunftsbedingte Kosten-Nutzen-Abwägung von Bildung kulturell bedingt zu differieren, wie die „positive Haltung gegenüber Bildung der Familie“ (S. 258) von türkischen Bildungsaufsteiger*innen zeigt (Tepecik, 2011). Der hohe Bildungswert in diesen Familien könnte auch den Blick auf Bildungsinvestitionen und damit das Kosten-Nutzen-Kalkül entgegen der eigentlichen Annahme für formal bildungsferne Familien verändern. Zusammengefasst zeigt sich also, dass Heranwachsende mit Migrationshintergrund unabhängig ihres Sozialstatus über einen hohen Leistungswillen (Schneider et al., 2017) und starke Bildungs- und Berufsambitionen (Relikowski et al., 2010) verfügen, die wiederum über die Einlösung des für die Eltern unerfüllt gebliebenen Bildungsversprechens befördert werden (Tepecik, 2011).

\footnotetext{
${ }^{2}$ Studierende mit hohem Sozialstatus erreichen die Hochschulreife häufiger am Gymnasium (Schneider et al., 2017). Driesel-Lange und Kracke (2017) konnten einen Entwicklungsbedarf berufswahlrelevanter Kompetenzen an eben dieser Schulform empirisch nachweisen.
} 


\section{Das Geschlecht als Faktor in der beruflichen Orientierung}

Die von Gottfredson (2002) theoretisch begründeten Geschlechterunterschiede lassen sich auch empirisch auf allen Ebenen der beruflichen Entwicklung nachzeichnen. Internationale Schulleistungsvergleiche zeigen Unterschiede in Form von erfolgreicheren schulischen Bildungsverläufe von Mädchen (OECD, 2015). Beispielhaft dafür ist auf internationaler Ebene die ausgeprägtere Lesekompetenz von Mädchen in allen PISA-Ländern (OECD, 2015), auf nationaler Ebene die besseren Abiturnoten der Mädchen (Schneider et al., 2017). In Folge der erfolgreicheren Schullaufbahnen erlangen Frauen im Vergleich zu Männern u. a. in Spanien, Belgien oder Finnland häufiger einen allgemeinbildenden oder beruflichen Abschluss auf Abiturniveau (OECD, 2015). Auch in Deutschland haben mehr Frauen als Männer eine Hochschulzugangsberechtigung ${ }^{3}$ (Autorengruppe Bildungsberichterstattung, 2018). Jungen und junge Männer scheinen zudem an größeren Übergangsschwierigkeiten zu leiden, die sich in ihrer größeren Repräsentanz im Übergangssektor widerspiegelt (Autorengruppe Bildungsberichterstattung, 2018).

Gleichzeitig nehmen nach wie vor weniger Frauen als Männer ein Hochschulstudium auf (Lörz et al., 2012; Schneider et al., 2017). Im Studium selbst sind Frauen im Durchschnitt jedoch erfolgreicher (Heublein \& Schmelzer, 2018). Die höheren Abbruchquoten der männlichen Studierenden lassen sich dabei nur zum Teil durch die differierende Studienfachwahl der beiden Geschlechter erklären (Heublein \& Schmelzer, 2018). Denn sowohl in den mathematischnaturwissenschaftlichen Studienfächern, in denen die männlichen Studierende überrepräsentiert sind, sowie den geisteswissenschaftlichen Fächern mit einem überproportional hohen Frauenanteil, brechen jeweils mehr junge Männer als Frauen ihr Bachelorstudium ab (Heublein \& Schmelzer, 2018). In der beruflichen Bildung zeigen sich ebenfalls geschlechtsbezogene Unterschiede. Weibliche Auszubildende verfügen häufiger über einen höheren Bildungsabschluss und durchlaufen ihre Ausbildung im Durchschnitt erfolgreicher als männliche Auszubildende (Senatsverwaltung für Gesundheit, Pflege und Soziales Berlin \& Amt für Statistik Berlin-Brandenburg, 2017, 2018). Der wissenschaftliche Diskurs erklärt diese leistungsbezogenen Geschlechterunterschiede anhand biologischer, gesellschafts- und schulsystemimmanenter Ursachen und ist in seinen Befunden entsprechend heterogen, wie Cremers (2012) feststellt.

Mädchen unterscheiden sich auch in ihrem Verhalten während des Berufswahlprozesses von Jungen. Sie beginnen früher mit der selbstbezogenen Exploration

\footnotetext{
${ }^{3}$ gemessen an der Altersgruppe der 20- bis 25-Jährigen
} 
(Driesel-Lange, 2011) und der gezielten Informationssuche zu Berufsausbildungen und Studienmöglichkeiten (Schneider et al., 2017). Trotz der früheren und intensiveren Exploration fühlen sich weibliche Jugendliche häufiger als männliche von der zu treffenden Entscheidung belastet (Fend, 1991). Diese erhöhte Unsicherheit führt bei ihnen entsprechend häufiger zu einer verzögerten Aufnahme nachschulischer Bildungsangebote, während männliche Heranwachsende am Übergang Schule-Hochschule - auch bei gleichen Leistungen und gleichem mathematischen Selbstkonzept - optimistischer auf das geplante Studium und ihre beruflichen Chancen schauen (Schneider et al., 2017; Schuchart, Keßler, Scheidt \& Buchwald, 2016).

Auch in Hinblick auf die Motive, eine Hochschulzugangsberechtigung zu erlangen, bestehen geschlechtsbezogene Unterschiede: Für junge Männer haben der Einfluss der Familie, die mit der Studienberechtigung assoziierte höhere Allgemeinbildung, sowie der berufliche Aufstieg und die gesellschaftliche Anerkennung eine höhere Bedeutung als für die jungen Frauen. Entsprechend nennen sie auch häufiger als weibliche Studierende ökonomische oder karrierebezogene Motive, wie Leistungs- und Führungsanspruch (Schneider et al., 2017). Im internationalen Vergleich sind hinsichtlich der beruflichen Ambitionen die Vorzeichen genau umgedreht: Mädchen streben in allen betrachteten Ländern, außer in China, Deutschland und der Schweiz signifikant häufiger als Jungen eine Karriere in einer Management- oder Expertenposition an (OECD, 2015). Diese Ergebnisse aus den PISA-Studien zeigen die im Kontext beruflicher Orientierung eingeschränkte Transfermöglichkeit internationaler Studienergebnisse auf. Daraus ergibt sich folglich eine erhöhte Relevanz für die Betrachtung des Berufswahlverhaltens von Jugendlichen im deutschsprachigen Raum, um den spezifischen kulturellen Kontext in seinem Einfluss abbilden zu können.

Steinritz, Lehmann-Grube und Ziegler (2016) konnten anhand geschlechtsund prestigebezogener Berufsbewertungen signifikante Unterschiede in den beruflichen Aspirationen von Jungen und Mädchen feststellen. Beide Geschlechter orientieren sich entsprechend Gottfredsons Berufswahltheorie anhand der ihnen entsprechenden Geschlechtskonnotation der Berufe. Mädchen wiesen jedoch ein größeres Aspirationsfeld mit einem breiteren geschlechtsbezogenen Akzeptanzbereich und einem geringeren Prestigeanspruch auf (Steinritz et al., 2016). Die geschlechtsspezifische Entwicklung beruflicher Interessensfelder lässt sich empirisch bereits für die (frühe) Adoleszenz nachweisen (Hoff, Briley, Wee \& Rounds, 2018; Pässler \& Schneider, 2019). Dabei schlagen sich die geschlechtsspezifischen Interessen bereits in der schulischen Leistungskurswahl der Sekundarsstufe II nieder: Mädchen wählen häufiger Kurse aus dem sprachlich-literarischen 
Bereich, während Jungen in den MINT-Fächern überrepräsentiert sind (DrieselLange \& Hany, 2005; Ministerium für Schule und Bildung des Landes NordrheinWestfalen, 2019b).

Die geschlechtstypbezogene Orientierung nach Gottfredson (1981) zeigt sich weiter in der Wahl der Ausbildungsberufe und Studiengänge. Die beliebtesten Ausbildungsberufe der weiblichen Auszubildenden sind dem Gesundheits-, Pflege- bzw. Erziehungsbereich, sowie dem betriebswirtschaftlichen Sektor zuzuordnen (Initiative Klischeefrei, 2018c; Senatsverwaltung für Gesundheit, Pflege und Soziales Berlin \& Amt für Statistik Berlin-Brandenburg, 2018). Bei den männlichen Auszubildenden präsentiert sich ein ähnliches begrenztes Berufswahlspektrum, das sich jedoch auf die handwerklich-technischen und betriebswirtschaftlichen Berufe bezieht (Initiative Klischeefrei, 2018c; Senatsverwaltung für Gesundheit, Pflege und Soziales Berlin \& Amt für Statistik Berlin-Brandenburg, 2018). Auch bei den Studienfächern bevorzugen Frauen neben den Rechts- und Betriebswissenschaften Studiengänge im Bereich Humanmedizin, Gesundheitsund Geisteswissenschaften (Initiative Klischeefrei, 2018b). Analog zu den Ausbildungsberufen sind in den MINT-Studiengängen die männlichen Studierenden besonders stark vertreten (Initiative Klischeefrei, 2018b). Es finden sich empirische Belege dafür, dass die Wahl geschlechtsstereotypischer Berufe ein Resultat unterschiedlicher Rollenverständnisse (Schmude, 2010) oder einer als hoch empfundenen Bedrohung der eigenen Geschlechtsidentität sein kann (Sinclair \& Carlsson, 2013). Auch die Bestärkung durch das soziale Umfeld (eher bei jungen Frauen), berufsbezogene Informationsdefizite (eher bei jungen Männern) (Haunberger \& Bartelsen, 2019) und ein differierender Leistungswillen (Hurrelmann, 2009) spielen eine Rolle. Die Ursachen können entsprechend Gottfredsons Berufswahltheorie (1981) als auch über die Sozialisierungstheorie nach Bourdieu (1982), im Sinne eines Geschlechterhabitus, eingeordnet und interpretiert werden.

Die geschlechtsbezogenen Unterschiede beruflicher Präferenzen manifestieren sich auch im weiteren beruflichen Verlauf, u. a. in einer nach wie vor geringeren Erwerbstätigenquote der Frauen ${ }^{4}$, in einem für sie ungünstigeren Verhältnis zwischen bezahlter und unbezahlter Arbeit (Initiative Klischeefrei, 2018a) sowie einer geschlechtsspezifischen Lohnlücke zu ungunsten der Frauen (Statistisches Bundesamt, 2016). Betont werden soll an dieser Stelle, dass die Gehaltsunterschiede nicht einzig den gewählten Berufsfeldern geschuldet sind. Vielmehr soll darauf hingewiesen werden, dass die sich bereits in der Kindheit entwickelnden,

\footnotetext{
${ }^{4}$ Dabei ist darauf hinzuweisen, dass sich der Anteil der erwerbstätigen Frauen in Westdeutschland seit 1991 von $58 \%$ auf knapp $74 \%$ gesteigert und damit der Erwerbstätigenquote der Frauen in Ostdeutschland (77\%) weitestgehend genähert hat (Bundesagentur für Arbeit, 2019, S. 69).
} 
auf Geschlechterattributionen basierenden Präferenzen langfristige und existientielle Konsequenzen bergen (Driesel-Lange, 2017) und daraus spezifische Bedarfe für die berufliche Orientierung von Mädchen und jungen Frauen resultieren.

In Hinblick auf die berufswahlbezogene Heterogenität und die Vielfalt in den Laufbahnverläufen lassen sich jedoch die Faktoren sozialer und kultureller Herkunft sowie das Geschlecht nicht ausschließlich separat voneinander betrachten, sondern müssen, wie in den theoretischen Betrachtungen von Gottfredson (2002), ebenso in ihrer Intersektionalität untersucht werden. Beispielhaft dafür sollen Beicht und Walden (2019) genannt werden, die beim Übergang nichtstudienberechtigter Schulabgänger*innen in den Ausbildungsmarkt ,ausgeprägte Wechselwirkungen“ (S. 53) zwischen dem Geschlecht, der sozialen Schicht und dem Migrationshintergrund festgestellt haben. Ihre Ergebnisse zeigen zum einen die Vielschichtigkeit in der Heterogenität der Bedarfe und unterstreichen zum anderen die Notwendigkeit, auch die Wirkweisen von Unterstützungsangeboten in der beruflichen Orientierung hinsichtlich dieser Heterogenität ausdifferenzierter zu betrachten.

\subsection{Zusammenfassung und Fazit}

Das Kapitel leitete das der Arbeit zugrunde liegende Verständnis von Heterogenität her (vgl. Teilkapitel 3.1). Es zeigte sich, dass verschiedene Definitionen, ähnlich dem im vorangegangenen Abschnitt (2.1) erläuterten Verständnis beruflicher Orientierung, aufgrund verschiedener Perspektiven bestehen (Scharenberg, 2013). Zudem lässt sich konstatieren, dass für das Verständnis von Heterogenität theoretische Überschneidungen mit den Begriffen der Inklusion und Diversity existieren (Plößner, 2013). Dabei kann Inklusion in Abgrenzung der drei Begriffe als die Summe der Bemühungen zur Ansprache heterogener Entwicklungsbedarfe spezifiziert werden (Grosche, 2015), während Diversity einen stärkeren Fokus auf die Einstellung zu den bestehenden Unterschieden der Individuen unternimmt (Plößner, 2013). Weiter wurde deutlich, dass Heterogenität immer in Beziehung zu Homogenisierung und Individualität gestellt werden muss, da diese sich in ihren Zielen und Wirkung gegenseitig bedingen und beeinflussen (Budde, 2015). In Anlehnung an die von Scharenberg (2013) für die empirische Bildungsforschung vorgeschlagene Definition, kann Heterogenität im Kontext beruflicher Orientierung als die Zusammenfassung aller Merkmale, über die sich Jugendliche in ihrer beruflichen Entwicklung unterscheiden, verstanden werden. 
Des Weiteren wurden in diesem Kapitel die aufgrund individueller Merkmale bestehenden Unterschiede in den Bildungs- und Übergangswahrscheinlichkeiten sowie im Berufswahlverhalten junger Menschen beleuchtet. Dabei wurde zur theoretischen Betrachtung der Ursachen und Wirkweisen dieser individuellen Faktoren zwischen primären und sekundären Herkunftseffekten unterschieden (vgl. Teilkapitel 3.2; Boudon, 1974), Unterschiede aufgrund prestige- und geschlechtsbezogener Selbst- und Berufsbilder fokussiert (Gottfredson, 1981) sowie milieugebundener Habitus beleuchtet (Bourdieu, 1982).

Boudon (1974) leitet theoretisch her, wie die soziale und kulturelle Herkunft die Bildungs- und Berufserfolge über zur Verfügung stehende Ressourcen direkt bedingen (Primäreffekte). Diese Effekte konnten hinsichtlich der Schulleistungen (Klieme et al., 2010; OECD, 2014; Stanat et al., 2002), der Ausbildungsund Studienwahrscheinlichkeiten (BIBB, 2019; Beicht \& Walden, 2019) sowie deren Erfolgswahrscheinlichkeiten (Heublein et al., 2017) empirisch nachgewiesen werden (vgl. Teilkapitel 3.3). Weiter wurde dargelegt, wie nach Boudon (1974) die individuell rationalen Bildungs- und Berufsentscheidungen durch schichtspezifische Kosten-Nutzen-Abwägungen und die zwischen der jeweiligen Herkunftsfamilie und einem Beruf existierende soziale Distanz beeinflusst werden (vgl. auch Brändle \& Grundmann, 2013). Diese Sekundäreffekte lassen sich jedoch nur bedingt nachweisen, u. a. in Hinblick auf die herkunftsabhängigen Unsicherheiten und Erfolgseinschätzung (Schneider et al., 2017). Mit Blick auf den kulturellen Hintergrund zeigt sich eine diffuse Befundlage. Denn die Effekte einer sozial bedingt schlechteren Kosten-Nutzen-Einschätzung von Bildung ließen sich für Heranwachsenden mit Migrationshintergrund aus sozialen Unterschichten nur eingeschränkt nachweisen (Tepecik, 2011). Weiter verfügen die jungen Migrant*innen unabhängig ihrer sozialen Herkunft über einen starken Leistungswillen (Schneider et al., 2017) und streben eine Statusverbesserung über monetär lukrative Berufe an (Schneider et al., 2017). Zum anderen zeigte sich bei ihnen im Gegensatz zu autochthonen sozial benachteiligten Heranwachsenden keine Relevanz der sozialen Distanz für Bildungsentscheidungen (Relikowski et al., 2010).

Auch Bourdieu (1982) setzt mit seinem Habitus, der das Erlernen und Erwerben von milieubedingten Denk- und Verhaltensweisen zusammenfasst (Scherr, 2016), die individuell rationalen Bildungs- und Berufsentscheidungen in den Kontext der jeweiligen Sozialisation (vgl. Brändle \& Grundmann, 2013). In der Anwendung des Habituskonzepts auf die berufliche Orientierung wurde deutlich, dass auch die beruflichen Entscheidungen der Heranwachsenden unbewusst durch die eigene Sozialisierung beeinflusst und die berufliche Orientierung freiwillig, aber unbewusst auf Berufe, die innerhalb des eigenen sozialen Milieus 
angesiedelt sind, beschränkt wird (vgl. Brändle \& Grundmann, 2013). Anhand von Gottfredsons entwicklungspsychologischer Theorie zur Eingrenzung und Kompromissbildung (1981, 1996, 2002) wurde darüber hinaus deutlich, wie Heranwachsende im Rahmen ihrer geschlechtsbezogenen und sozialen Identitätsbildung einen Abgleich des eigenen Selbstbilds mit Berufsbildern vornehmen und dabei Berufe frühzeitig noch vor einem Abgleich von Interessen und Tätigkeiten für sich ausschließen.

Alle drei Theorien verdeutlichen zudem, dass die abgebildeten Mechanismen der beruflichen Entscheidungen zwar individuell rational erscheinen, jedoch durch darüber liegende soziale Strukturen und die eigene Sozialisierung eingegrenzt und gesteuert werden (vgl. Brändle \& Grundmann, 2013). Der bereits vielfach artikulierte Bedarf frühzeitiger Sensibilisierung der Jugendlichen einerseits, sowie der beteiligten Akteur*innen andererseits lässt sich dadurch nur bestätigen (Faulstich-Wieland \& Scholand, 2017).

Hinsichtlich des Geschlechts zeigen die vorgestellten empirischen Befunde klare Unterschiede in den beruflichen Eingrenzungsprozessen (Driesel-Lange, 2011; Haunberger \& Bartelsen, 2019; Steinritz et al., 2016). Aus diesen Unterschieden lassen sich differierende Unterstützungsbedarfe in der beruflichen Orientierung ableiten. Während Jungen aufgrund ihrer später einsetzenden Exploration (Driesel-Lange, 2011) von frühzeitigen, explorationsfördernden Impulsen profitieren könnten, benötigen Mädchen ggfs. mehr Zuspruch und Unterstützung beim Abbau von Unsicherheiten (Schuchart et al., 2016). Ziel von berufsorientierenden Maßnahmen ist es, Jugendliche in ihrer beruflichen Orientierung zu unterstützen und entsprechend ihrer Bedarfe zu adressieren (KMK, 2017b). Im Sinne „evidenzbasierter Berufsorientierung“ (Brüggemann, 2015, S. 77), wird durch die Erkenntnisse geschlechterspezifischer Präferenzen auch der Bedarf deutlich, berufsorientierende Angebote in Hinblick auf geschlechtsspezifische Effekte hin zu überprüfen.

Jedoch bleibt die Befundlage zu geschlechterbezogenen Unterschieden in der Maßnahmennutzung und -wirkung spärlich, sodass die Untersuchung von Geschlechterunterschieden in der Wahrnehmung berufsorientierender Maßnahmen weiterhin ein Forschungsdesiderat darstellt (Driesel-Lange \& Ohlemann, 2019). Dieses wird durch die empirische Studie in Kapitel 9 aufgenommen. Unberücksichtigt bleibt in den vorgelegten Studien zudem häufig die Beziehung der einzelnen Faktoren zueinander und deren intersektionale Effekte auf die berufliche Orientierung. Hadjar und Hupka-Brunner (2013) zeigen die Notwendigkeit einer systematischeren Betrachtung intersektionaler Wirkmechanismen auf. Die wenigen bestehenden Analysen zu intersektionalen Effekten persönlicher Merkmale im Kontext beruflicher Entwicklung und Einmündung (Beicht \& Walden, 2019; 
Busch, 2013; Schmude, 2010) lassen auf eine Fragmentierung der Heranwachsenden in viele Untergruppen mit differierenden Bedarfen schließen. Inwieweit diese intersektionalen Zusammenhänge sich auch für die Effekte berufsorientierender Maßnahmen zeigen, bleibt zu untersuchen. Die empirische Studie in Kapitel 9 widmet sich entsprechend auch dieser benannten Forschungslücke und analysiert die zielgruppenabhängigen, intersektionalen Effekten berufsorientierender Maßnahmen. Die Berücksichtigung differierender Entwicklung kann über die Anpassung der Konzepte und Programme berufliche Orientierung an individuelle Bedarfe, also über ihre Individualisierung, geschehen. Scharenberg (2013) spricht hier auch von Homogenisierung der Gruppen. Der Identifizierung homogener Gruppen zur Binnendifferenzierung widmet sich die in Kapitel 11 vorgestellte Studie.

Die theoretischen Konzepte und empirischen Befunde zusammenfassend werden Bildungs- und Berufskarrieren durch das Geschlecht sowie die soziale und kulturelle Herkunft der Heranwachsenden direkt und indirekt beeinflusst. Des Weiteren lassen sich intersektionale Effekte der individuellen Merkmale auf das Berufswahlverhalten beobachten, die auch in der Beforschung von berufsorientierenden Maßnahmen Berücksichtigung finden müssen. Wird im Anschluss daran auf praktische Implikationen fokussiert, so können Maßnahmen der beruflichen Orientierung bedarfsgerecht gestaltet und adressiert werden.

Neben den benannten personenbezogenen, die Berufswahl beeinflussenden Voraussetzungen, kann die Heterogenität der Bedarfe auch anhand immanenter berufswahlbezogener Entwicklungsprozesse erklärt werden. Das folgende Kapitel präsentiert und vergleicht ausgewählte theoretische Modelle, die aus unterschiedlichen Perspektiven berufswahlbezogene Phasen beschreiben, um die Phasen als Quelle heterogener Bedarfe zu verdeutlichen. 
Open Access Dieses Kapitel wird unter der Creative Commons Namensnennung 4.0 International Lizenz (http://creativecommons.org/licenses/by/4.0/deed.de) veröffentlicht, welche die Nutzung, Vervielfältigung, Bearbeitung, Verbreitung und Wiedergabe in jeglichem Medium und Format erlaubt, sofern Sie den/die ursprünglichen Autor(en) und die Quelle ordnungsgemäß nennen, einen Link zur Creative Commons Lizenz beifügen und angeben, ob Änderungen vorgenommen wurden.

Die in diesem Kapitel enthaltenen Bilder und sonstiges Drittmaterial unterliegen ebenfalls der genannten Creative Commons Lizenz, sofern sich aus der Abbildungslegende nichts anderes ergibt. Sofern das betreffende Material nicht unter der genannten Creative Commons Lizenz steht und die betreffende Handlung nicht nach gesetzlichen Vorschriften erlaubt ist, ist für die oben aufgeführten Weiterverwendungen des Materials die Einwilligung des jeweiligen Rechteinhabers einzuholen.

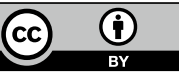

